\title{
A note on the shape of the probability weighting function
}

\author{
Martina Nardon and Paolo Pianca
}

\begin{abstract}
The focus of this contribution is on the transformation of objective probability, which in Prospect Theory is commonly referred as probability weighting. Empirical evidence suggests a typical inverse-S shaped function: decision makers tend to overweight small probabilities, and underweight medium and high probabilities; moreover, the probability weighting function is initially concave and then convex. We apply different parametric weighting functions proposed in the literature to the evaluation of derivative contracts and to insurance premium principles.
\end{abstract}

\section{Introduction}

Cumulative Prospect Theory (CPT) has been proposed in [7] as an alternative to Expected Utility to explain actual behaviors. Formally, CPT relies on two key transformations: the value function $v$, which replaces the utility function for the evaluation of outcomes, and a distortion function for objective probabilities $w$, which models probabilistic risk behavior. Risk attitudes are derived from the shapes of these functions as well as their interaction. The focus of this contribution is on the transformation of objective probability, which is commonly referred as probability weighting or probability distortion.

A weighting function $w$ is a strictly increasing function which maps the probability interval $[0,1]$ into $[0,1]$, with $w(0)=0$ and $w(1)=1$. Evidence suggests a typical inverse-S shape: small probabilities are overweighted, $w(p)>p$, whereas medium and high probabilities are underweighted, $w(p)<p$. The curvature of the weighting

Martina Nardon

Ca' Foscari University of Venice, Department of Economics, San Giobbe - Cannaregio 873, 30121 Venezia, Italy, e-mail: mnardon@unive.it

Paolo Pianca

Ca' Foscari University of Venice, Department of Economics, San Giobbe - Cannaregio 873, 30121

Venezia, Italy e-mail: pianca@unive.it 
function is related to the risk attitude towards probabilities; the function is initially concave (probabilistic risk seeking or optimism) for probabilities in the interval $\left(0, p^{*}\right)$, and then convex (probabilistic risk aversion or pessimism) in the interval $\left(p^{*}, 1\right)$, for a certain value of $p^{*}$. A linear weighting function describes probabilistic risk neutrality or objective sensitivity towards probabilities, which characterizes Expected Utility. Empirical findings indicate that the intersection (elevation) between the weighting function and the 45 degrees line, $w(p)=p$, is for $p^{*}$ in the interval $(0.3,0.4)$. Figure 1 shows instances with different elevation and curvature of the two parameter constant relative sensitivity probability weighting function proposed by [1].

Different parametric forms for the weighting function with the above mentioned features have been proposed in the literature, and their parameters have been estimated in many empirical studies. Some forms are derived axiomatically or are based on psychological factors. Single parameter and two (or more) parameters weighting functions have been suggested; some functions have linear, polynomial or other forms, and there is also some interest for discontinuous weighting functions. Two commonly applied weighting functions are those proposed by Tversky and Kahneman [7] $w(p)=\frac{p^{\gamma}}{\left(p^{\gamma}+(1-p)^{\gamma}\right)^{1 / \gamma}}$, with $w(0)=0$ and $w(1)=1$, and $\gamma>0$ (with some constraint in order to have an increasing function); and Prelec [5] $w(p)=e^{-\delta(-\ln p)^{\gamma}}$, with $w(0)=0$ and $w(1)=1,0<\delta<1, \gamma>0$. When $\gamma<1$, one obtains the inverse-S shape.

The choice of the probability weighting function should be driven by the following motivations: its empirical properties, intuitive and empirically testable preference conditions, nonlinear behavior of the probability weighting function. Moreover, a parametric probability weighting function should be parsimonious (remaining consistent with the properties suggested by empirical evidence), in particular when we consider different parameters for the weighting of probability of gains and losses.

We analyze some applications in finance to the evaluation of derivative contracts (see [4]) and in insurance to premium principles, briefly discussed in the next section.

\section{An application to premium calculation}

Let $u$ denote the utility function, and $W$ be the initial wealth; the utility indifference price $P$ is the premium from the insurer's viewpoint which satisfies (if it exists) the condition:

$$
u(W)=\mathbb{E}[u(W+P-X)],
$$

where $X$ is the possible loss, modeled with a non-negative continuous random variable. The premium $P$ makes indifferent the insurance company about accepting the risky position and not selling the insurance policy. 

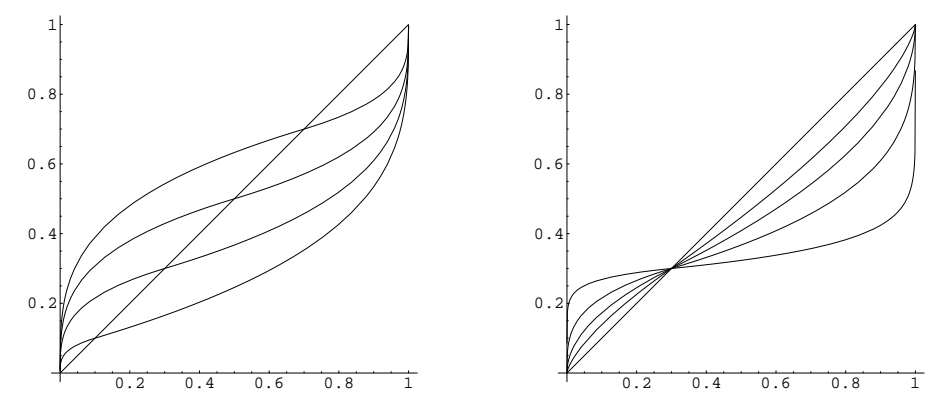

Fig. 1 Instances of the two parameter probability weighting function proposed by [1], with different elevation (left) and curvature (right).

Differently from Expected Utility, in Prospect Theory individuals are risk averse when considering gains and risk-seeking with respect to losses; moreover, they are more sensitive to losses than to gains of comparable magnitude (loss aversion). The final result $W+P-X$ in (1) could be positive or negative. Results are evaluated considering potential gains and losses relative to a reference point, rather than in terms of final wealth, hence assuming zero as reference point (the status quo), the relative result $P-X$ will be considered. Decision weights are differences in transformed cumulative probabilities of gains or losses.

We consider the cumulative prospect value for a continuous random variable [2]:

$$
V=\int_{-\infty}^{0} \Psi^{-}[F(x)] f(x) v^{-}(x) d x+\int_{0}^{+\infty} \Psi^{+}[1-F(x)] f(x) v^{+}(x) d x
$$

where $\Psi=\frac{d w(p)}{d p}$ is the derivative of the weighting function $w, F$ is the cumulative distribution function and $f$ is the probability density function of the outcomes.

Condition (1) under continuous CPT becomes (see also [3]):

$$
0=\int_{-\infty}^{0} \Psi^{-}[F(x)] f(x) v^{-}(P-x) d x+\int_{0}^{+\infty} \Psi^{+}[1-F(x)] f(x) v^{+}(P-x) d x
$$

We also assume that decision makers are not indifferent among frames of cash flows: the framing of alternatives exerts a crucial effect on actual choices. People may keep different mental accounts for different types of outcomes, and when combining these accounts to obtain overall result, typically they do not simply sum up all monetary amounts, but intentionally use hedonic framing [6] such that the combination of the outcomes appears more favorable and increases their utility. Outcomes are aggregated or segregated depending on what leads to the highest possible prospect value: multiple gains are preferred to be segregated (narrow framing), losses are preferred to be integrated with other losses (or large gains) in order to ease the pain of the loss. Mixed outcomes would be integrated in order to cancel out losses when there is a net gain or a small loss; for large losses and a small gain, 
they usually are segregated in order to preserve the silver lining. This is due to the shape of the value function in Prospect Theory, characterized by risk-seeking or risk aversion, diminishing sensitivity and loss aversion.

If we segregate the cashed premium from the possible loss, condition (3) becomes

$$
0=v^{+}(P)+\int_{-\infty}^{0} \Psi^{-}[F(x)] f(x) v^{-}(-x) d x .
$$

A usual choice for the value function is

$$
\begin{cases}v^{-}=-\lambda(-x)^{b} & x<0 \\ v^{+}=x^{a} & x \geq 0\end{cases}
$$

which leads to the following result:

$$
P=\left(\lambda \int_{0}^{+\infty} \Psi^{-}[F(x)] f(x) x^{b} d x\right)^{1 / a} .
$$

Alternative functional forms both for the value function and the probability weighting function, embedded in CPT framework (3), yield a different model with potentially different implications for choice behavior. In particular, when the weighting function has an inverse-S shape, very low probability of extreme events are overweighted. We apply different probability weighting functions and study the effect on the premium calculation. In particular, two parameters allow for separate control of curvature and elevation, and the constant relative sensitivity probability weighting function proposed by [1], which models distinctly these two features, is of particular interest.

\section{References}

1. Abdellaoui, M., L'Haridon, O., Zank, H.: Separating curvature and elevation: A parametric probability weighting function. J. Risk Uncertain. 41, 39-65 (2010)

2. Davies, G.B., Satchell, S.E.: The behavioural components of risk aversion. J. Math. Psychol. 51, 1-13 (2007)

3. Kaluszka, M., Krzeszowiec, M.: Pricing insurance contracts under Cumulative Prospect Theory. Insurance: Math. Econ. 50, 159-166 (2012)

4. Nardon, M., Pianca, P.: A behavioural approach to the pricing of European options. In: Corazza, M., Pizzi, C. (eds) Mathematical and Statistical Methods for Actuarial Sciences and Finance, pp. 217-228. Springer-Verlag, Milano (2014)

5. Prelec, D.: The probability weighting function. Econom. 66, 497-527 (1998)

6. Thaler, R.H.: Mental accounting and consumer choice. Mark. Science. Summer, 199-214 (1985)

7. Tversky, A., Kahneman, D.: Advances in prospect theory: cumulative representation of the uncertainty. J. Risk. Uncertain. 5, 297-323 (1992) 